ARTICLE

Received 13 Feb 2014 | Accepted 30 Apr 2014 | Published 30 May 2014 DOl: 10.1038/ncomms5016

\title{
High-efficiency organic light-emitting diodes with fluorescent emitters
}

Hajime Nakanotani,2, Takahiro Higuchi', Taro Furukawa1, Kensuke Masui ${ }^{1,3}$, Kei Morimoto1, Masaki Numata1, Hiroyuki Tanaka1, Yuta Sagara1, Takuma Yasuda1,4 \& Chihaya Adachi1,2,4

Fluorescence-based organic light-emitting diodes have continued to attract interest because of their long operational lifetimes, high colour purity of electroluminescence and potential to be manufactured at low cost in next-generation full-colour display and lighting applications. In fluorescent molecules, however, the exciton production efficiency is limited to $25 \%$ due to the deactivation of triplet excitons. Here we report fluorescence-based organic light-emitting diodes that realize external quantum efficiencies as high as $13.4-18 \%$ for blue, green, yellow and red emission, indicating that the exciton production efficiency reached nearly $100 \%$. The high performance is enabled by utilization of thermally activated delayed fluorescence molecules as assistant dopants that permit efficient transfer of all electrically generated singlet and triplet excitons from the assistant dopants to the fluorescent emitters. Organic light-emitting diodes employing this exciton harvesting process provide freedom for the selection of emitters from a wide variety of conventional fluorescent molecules.

\footnotetext{
${ }^{1}$ Center for Organic Photonics and Electronics Research (OPERA), Kyushu University, 744 Motooka, Nishi, Fukuoka 819-0395, Japan. ${ }^{2}$ Innovative Organic Device Laboratory, Institute of Systems, Information Technologies and Nanotechnologies (ISIT), 744 Motooka, Nishi, Fukuoka 819-0395, Japan. ${ }^{3}$ Advanced Research Laboratories, Fujifilm Co., 577 Ushijima, Kaisei, Ashigarakami, Kanagawa 258-8577, Japan. ${ }^{4}$ International Institute for Carbon Neutral Energy Research (WPI-I2CNER), Kyushu University, 744 Motooka, Nishi, Fukuoka 819-0395, Japan. Correspondence and requests for materials should be addressed to C.A. (email: adachi@cstf.kyushu-u.ac.jp).
} 
S pin statistics states that one singlet exciton is generated for every three triplet excitons after the recombination of holes and electrons in organic semiconductor materials ${ }^{1}$. Light emission can be extracted through fluorescence or phosphorescence by the decay of these excitons from a singlet $\left(\mathrm{S}_{1}\right)$ or triplet $\left(\mathrm{T}_{1}\right)$ excited state to a ground state. By utilizing efficient radiative decay of electrically generated excitons, organic light-emitting diodes (OLEDs) are attracting intense attention for use as advanced displays and lighting sources.

The external electroluminescence (EL) quantum efficiency $\left(\eta_{\mathrm{EQE}}\right)$ of OLEDs is a key parameter and is described by the wellknown equation

$$
\eta_{\mathrm{EQE}}=\eta_{\text {int }} \times \eta_{\text {out }}=\left(\gamma \times \eta_{\gamma} \times \Phi_{\mathrm{PL}}\right) \times \eta_{\text {out }}
$$

where $\eta_{\text {int }}$ is the internal EL quantum efficiency and $\eta_{\text {out }}$ is the light-out-coupling efficiency. According to equation (1), $\eta_{\text {int }}$ is limited by the following three factors: (i) charge balance of injected holes and electrons $(\gamma)$, (ii) efficiency of radiative exciton production $\left(\eta_{\gamma}\right)$ and (iii) photoluminescence (PL) quantum yield of the emitter molecules $\left(\Phi_{\mathrm{PL}}\right)$. The ideal $\gamma$ can be achieved by circumspect design of OLED structures with the appropriate selection of charge transport layers, host-guest system, and anode and cathode materials. In addition, based on a proper molecular design for light emission, $\Phi_{\mathrm{PL}}$ of nearly $100 \%$ has been demonstrated in a wide variety of fluorescent and phosphorescent materials. However, $\eta_{\gamma}$ can severely limit $\eta_{\text {int }}$ if the $75 \%$ of electrically generated excitons formed in triplet states are not harvested.

Several routes have been proposed to obtain a high $\eta_{\text {int }}$ through the efficient harvesting of triplet excitons in OLEDs. While the very first trials of ketone derivatives showing intense phosphorescence at low temperature opened a new method for triplet harvesting, the $\eta_{\mathrm{EQE}}$ was limited to a low value ${ }^{2}$. Rare metal complexes containing $\mathrm{Eu}$ and $\mathrm{Tb}$ established intramolecular cascade energy transfer as another route to harvest triplet excitons but did not show promising $\eta_{\mathrm{EQE}}{ }^{3-5}$. Later, a successful strategy was realized using room temperature phosphorescent emitters such as platinum and iridium complexes. In this case, according to the mixing of the spin orbitals of $S_{1}$ and $T_{1}$ states due to the presence of a heavy atom, the radiative decay rate from a $T_{1}$ state to a ground state is significantly accelerated, resulting in the radiative decay of nearly $100 \%$ of triplet excitons ${ }^{6-10}$. In addition, the utilization of phosphorescence emitters as a triplet sensitizer has been proposed ${ }^{11,12}$. Using this process, triplet harvesting realized by energy transfer from the $\mathrm{T}_{1}$ state of a phosphorescent emitter such as an iridium 2-phenylpyridine complex to the $S_{1}$ state of a fluorescent emitter via dipole-dipole coupling (that is, Förster energy transfer, FRET) resulted in an $\eta_{\text {int }}$ of $\sim 45 \%$ (ref. 12). However, the rather limited $\eta_{\text {int }}$ is due to the presence of the competitive deactivation process of triplet-triplet energy transfer.

Although OLEDs based on fluorescent molecules, which are composed of simple aromatic compounds, have continued to attract interest because of their longer operational lifetimes in blue OLEDs, higher colour purity (narrow spectral width) EL and broader freedom of molecular design compared with phosphorescence-based OLEDs ${ }^{13-15}$, the $\eta_{\text {int }}$ of traditional fluorescencebased OLEDs is limited to less than $25 \%$ even in the ideal case. Therefore, the enhancement of $\eta_{\text {int }}$ in OLEDs using conventional fluorescence-based emitters is still obviously a major concern for the development of future OLEDs.

To achieve this, singlet exciton generation via a triplet-triplet annihilation (TTA) process is one possible route ${ }^{16,17}$. However, the theoretical upper limit of the singlet exciton production ratio when the TTA process is included is still less than 62.5\%, corresponding to an $\eta_{\mathrm{EQE}}$ of $12.5 \%$ in the ideal case ${ }^{18}$. Recently, we demonstrated an alternative route for increasing $\eta_{\text {int }}$ by using a thermally activated delayed fluorescence (TADF) process ${ }^{19-23}$. In the TADF process, light emission can be extracted as delayed fluorescence after intersystem crossing (ISC) from the $\mathrm{T}_{1}$ to the $\mathrm{S}_{1}$ states in TADF emitters, resulting in efficient radiative decay from the $S_{1}$ state. In fact, after comprehensive development of TADF materials, an $\eta_{\text {int }}$ of nearly $100 \%$ was realized in green OLEDs ${ }^{21}$.

In addition to the use of the TADF process for emitters in OLEDs, we propose a promising route for triplet harvesting by applying TADF molecules as an assistant dopant in OLEDs. Thus, the OLED is composed of a double-dopant system-that is, a wide-energy-gap host, a TADF-assistant dopant and a fluorescent emitter dopant-that leads to $\eta_{\gamma}=100 \%$. In this system, triplet excitons created on an assistant TADF molecule $\left(\mathrm{T}_{1}^{\mathrm{A}}\right.$ ) by electrical excitation are upconverted to the $\mathrm{S}_{1}$ state of the TADF molecule $\left(S_{1}^{A}\right)$, and all $S_{1}^{A}$ excitons are transferred to the $S_{1}$ state of a fluorescent emitter molecule $\left(S_{1}^{\mathrm{E}}\right)$ via a FRET process, which results in efficient radiative decay from $S_{1}^{E}$ of the fluorescent emitter.

On the basis of this cascade energy transfer, we demonstrate highly efficient OLEDs with $\eta_{\mathrm{EQE}}$ as high as 13.5, 15.8, 18 and $17.5 \%$ for blue, green, yellow and red colours, respectively. We use the TADF molecules 10-phenyl-10 $\mathrm{H}, 10^{\prime} \mathrm{H}$-spiro[acridine-9, $9^{\prime}$-anthracen]-10'-one (ACRSA $)^{23}$, 3-(9,9-dimethylacridin-10 (9H)-yl)-9H-xanthen-9-one (ACRXTN), 2-phenoxazine-4, 6-diphenyl-1,3,5-triazine (PXZ-TRX $)^{20}$ and 2,4,6-tri $(4-(10 \mathrm{H}-$ phenoxazin-10H-yl)phenyl)-1,3,5-triazine (tri-PXZ-TRZ) ${ }^{22}$ as assistant dopants and 2,5,8,11-tetra-tert-butylperylene (TBPe), 9,10-Bis[N,N-di-(p-tolyl)-amino]anthracene (TTPA), 2,8-ditert-butyl-5,11-bis(4-tert-butylphenyl)-6,12-diphenyltetracene (TBRb) and tetraphenyldibenzoperiflanthene (DBP) as fluorescent emitter dopants (Fig. 1a). These fluorescent molecules have been widely used as conventional fluorescent emitters in OLEDs and are commercially available ${ }^{24-27}$. As host materials, we use bis-(2-(diphenylphosphino)phenyl)ether oxide (DPEPO), 1,3-Bis $(\mathrm{N}$-carbazolyl)benzene (mCP), 3,3-di(9H-carbazol-9yl)biphenyl (mCBP) and 4,4'-bis(9-carbazolyl)-1,1'-biphenyl (CBP) for the blue, green, yellow and red OLEDs, respectively. In this double-dopant system, the assistant dopant does not itself emit light but passes all of the electrically generated excitons to fluorescent emitter molecules for radiative decay.

\section{Results}

Energy transfer process. Figure 1a shows an energy transfer diagram for the emitter layers (EMLs) in our cascade-type EL devices. The emitter and assistant dopant molecule combinations and concentrations studied as EMLs here are listed in Table 1. In the case of an EML without any assistant dopant, the injected carriers are transported on the host molecules, and the carriers are eventually trapped on an emitter dopant due to its shallower highest occupied molecular orbital and deeper lowest unoccupied molecular orbital compared with those of the host material. This results in direct carrier recombination dominantly on the emitter dopants. Therefore, no triplet excitons contribute to the total EL efficiency.

On the other hand, when assistant dopants are doped into these EMLs, exciton formation mainly on the assistant molecules is desired. Here to reduce the effect of direct carrier trapping on the emitter molecules in the EMLs, the doping concentration of the emitter dopants was held at $1 \mathrm{wt} \%$. Conversely, assistant dopants were doped into the EMLs in doping concentrations ranging between 15 and $50 \mathrm{wt} \%$, which ensures that the assistant dopants act as the main carrier recombination centres in the EMLs. Thus, after both singlet $\left(S_{1}^{A}\right)$ and triplet $\left(T_{1}^{A}\right)$ exciton 
a

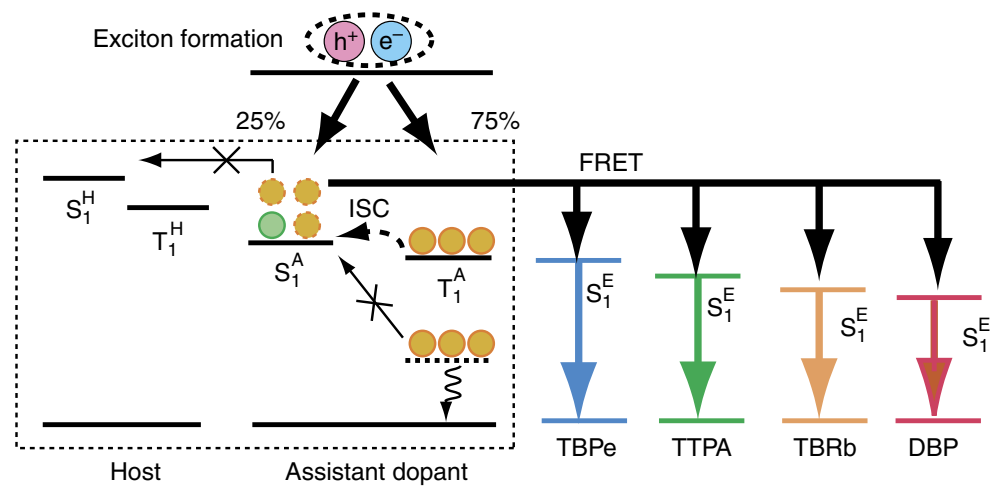

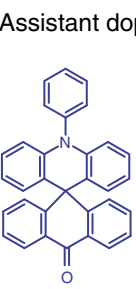

ACRSA

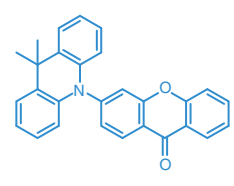

ACRXTN

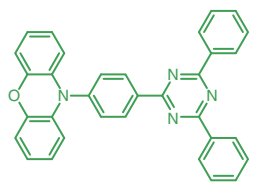

PXZ-TRZ

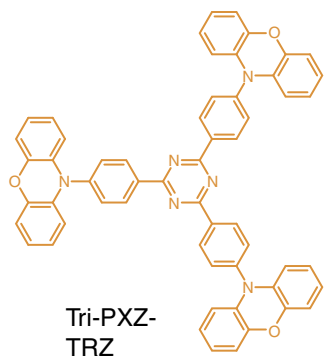

b

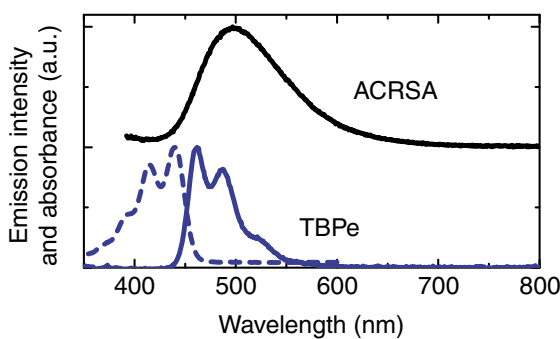

d

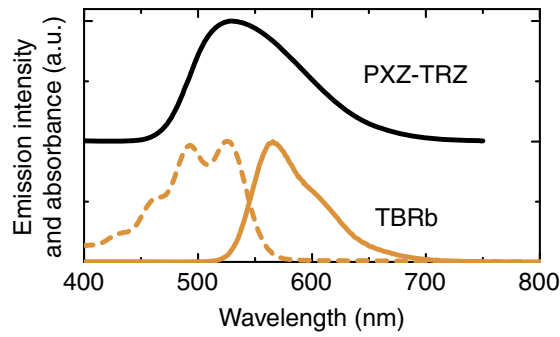

C

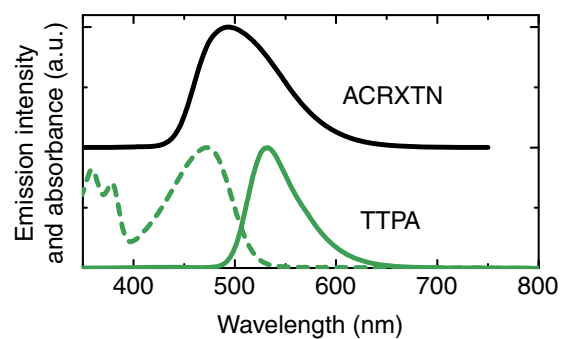

e

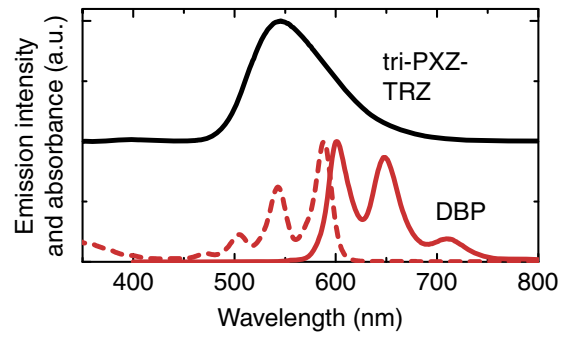

Figure 1 | Energy transfer mechanism. (a) Schematic illustration of proposed energy transfer mechanism in the emitter dopant:assistant dopant:host matrix under electrical excitation and chemical structures of the assistant dopants used in this study. (b-e) Fluorescence spectra of assistant dopant:host co-deposited film (upper), and absorption (dashed line) and fluorescence (solid line) spectra of emitter dopant in solution $\left(10^{-5} \mathrm{moll}^{-1}\right.$ in $\mathrm{CH}_{2} \mathrm{Cl}_{2}$ ) (bottom). Rather large Förster transfer radii of $\sim 2.2, \sim 7.3, \sim 6.9$ and $\sim 10 \mathrm{~nm}$ were estimated for blue, green, yellow and red EML matrices based on the spectral overlap between the absorption spectrum of the acceptor and the PL spectrum of the donor, suggesting that efficient FRET is possible.

formation on the assistant dopants, the formed triplet excitons are upconverted to the $S_{1}^{A}$ state through ISC because of the rather small energy gap between the $\mathrm{S}_{1}^{\mathrm{A}}$ and $\mathrm{T}_{1}^{\mathrm{A}}$ levels $\left(\Delta E_{\mathrm{ST}}\right)$, as summarized in Table 1 . Then, according to the spectral overlap between the absorption spectra of the emitter molecules and the PL spectra of the assistant dopants (Fig. 1b-e), the $S_{1}^{\mathrm{A}}$ exciton energies are resonantly transferred to the $S_{1}^{\mathrm{E}}$ states of emitter molecules based on a FRET process. Finally, light can be emitted as delayed fluorescence from the $S_{1}^{\mathrm{E}}$ state of the emitter molecules. If conventional fluorescence molecules were used as the assistant dopant, triplet excitons would non-radiatively decay from $\mathrm{T}_{1}^{\mathrm{A}}$ to the ground state and would not contribute to light emission because of the large $\Delta E_{\mathrm{ST}}$ of conventional fluorescence molecules as shown in Fig. 1a. Thus, the created triplet excitons would not contribute to the energy transfer processes if the assistant dopant is a conventional fluorescence material.

The proposed influence of concentration on carrier trapping and recombination is well supported by the dependence of $\eta_{\mathrm{EQE}}$ on the concentration of the assistant dopant as shown in Supplementary Figs 1-3 and Supplementary Note 1. Here we observed the best $\eta_{\mathrm{EQE}}$-luminance $(L)$ characteristics with the assistant dopant concentration of $15 \%$. In the case of assistant dopant concentrations less than $15 \%$, carrier recombination may not perfectly occur on the assistant dopants, while Dexter energy 
Table 1 | Components of the emitter layers of the four colour OLEDs.

\begin{tabular}{|c|c|c|c|c|c|c|c|}
\hline EL colour & $\begin{array}{c}\text { Host } \\
\left(S_{1}^{H}, T_{1}^{H}\right)^{\star}(e V)\end{array}$ & $\begin{array}{c}\text { Assistant } \\
\text { dopant } \\
\left(\mathrm{S}_{1}^{\mathrm{A}}, \mathrm{T}_{1}^{A}\right)^{\star}(\mathrm{eV})\end{array}$ & $\begin{array}{c}\text { Assistant dopant } \\
\text { concentration (wt\%) }\end{array}$ & $\begin{array}{l}\Delta E_{\mathrm{ST}} \\
(\mathrm{eV})\end{array}$ & $\begin{array}{c}\text { Emitter } \\
\text { dopant } \\
S_{1}^{E^{\star}}(e V)\end{array}$ & $\begin{array}{c}\text { Emitter dopant } \\
\text { concentration (wt\%) }\end{array}$ & $\Phi_{\mathrm{PL}}(\%)$ \\
\hline Blue & $\begin{array}{c}\text { DPEPO } \\
(3.50,3.00)\end{array}$ & $\begin{array}{c}\text { ACRSA } \\
(2.55,2.52)\end{array}$ & 15 & 0.03 & $\begin{array}{l}\text { TBPe } \\
(2.69)\end{array}$ & 1 & $80 \pm 2$ \\
\hline Green & $\begin{array}{c}\mathrm{mCP} \\
(3.40,2.90)\end{array}$ & $\begin{array}{c}\text { ACRXTN } \\
(2.53,2.47)\end{array}$ & 50 & 0.06 & $\begin{array}{l}\text { TTPA } \\
(2.34)\end{array}$ & 1 & $81 \pm 2$ \\
\hline Yellow & $\begin{array}{c}\mathrm{mCBP} \\
(3.37,2.90)\end{array}$ & $\begin{array}{c}\text { PXZ-TRZ } \\
(2.30,2.23)\end{array}$ & 25 & 0.07 & $\begin{array}{l}\text { TBRb } \\
(2.18)\end{array}$ & 1 & $90 \pm 2$ \\
\hline Red & $\begin{array}{c}\text { CBP } \\
(3.36,2.55)\end{array}$ & $\begin{array}{l}\text { tri-PXZ-TRZ } \\
(2.27,2,16)\end{array}$ & 15 & 0.11 & $\begin{array}{l}\text { DBP } \\
(2.03)\end{array}$ & 1 & $88 \pm 2$ \\
\hline
\end{tabular}

ACRSA, 10-phenyl-10H,10'H-spiro[acridine-9,9'-anthracen]-10'-one; ACRXTN, 3-(9,9-dimethylacridin-10(9H)-yl)-9H-xanthen-9-one; CBP, 4,4'-bis(9-carbazolyl)-1,1'-biphenyl; DBP,

tetraphenyldibenzoperiflanthene; DPEPO, bis-(2-(diphenylphosphino)phenyl)ether oxide; EL, electroluminescence; mCBP, 3,3-di(9H-carbazol-9-yl)biphenyl; mCP, 1,3-bis(N-carbazolyl)benzene; PXZ-

TRZ, 2-phenoxazine-4,6-diphenyl-1,3,5-triazine; OLED, organic light-emitting diode; TBPe, 2,5,8,11-tetra-tert-butylperylene; TBRb, 2,8-di-tert-butyl-5,11-bis(4-tert-butylphenyl)-6,12-diphenyltetracene; tri-

PXZ-TRZ, tri-PXZ-TRZ, 2,4,6-tri(4-(10H-phenoxazin-10H-yl)phenyl)-1,3,5-triazine; TTPA, 9,10-bis[N,N-di-(p-tolyl)-amino]anthracene.

${ }^{*}$ The $S_{1}$ and $T_{1}$ energies were estimated from the peak wavelengths of fluorescence and phosphorescence emission, respectively.

transfer from the assistant dopants to the triplet level of a fluorescent emitter $\left(\mathrm{T}_{1}^{\mathrm{E}}\right)$ or concentration quenching of the assistant dopants may happen in the case of the concentrations over $15 \%$. The optimum concentration would change for different combinations of host and guest molecules having different carrier transport and photophysical characteristics. We note that the host layers have both higher singlet $\left(S_{1}^{\mathrm{H}}\right)$ and triplet $\left(\mathrm{T}_{1}^{\mathrm{H}}\right)$ energy levels than those of the assistant molecules, $\mathrm{S}_{1}^{\mathrm{A}}$ and $\mathrm{T}_{1}^{\mathrm{A}}$. Therefore, back energy transfer from the $S_{1}^{A}$ state of the assistant dopants to the $\mathrm{S}_{1}^{\mathrm{H}}$ state of the host layers is inhibited. In addition, dispersing the assistant dopants in a host matrix can additionally prevent direct energy transfer from $\mathrm{T}_{1}^{\mathrm{A}}$ to $\mathrm{T}_{1}^{\mathrm{E}}$-that is, minimize a Dexter energy transfer process that would result in losses ${ }^{11}$.

OLED characteristics. On the basis of the optimized configuration of EMLs for blue, green, yellow and red EL, full-colour OLEDs were fabricated to demonstrate the impact of this triplet harvesting process on OLED performance. The $\eta_{\mathrm{EQE}^{-}} L$ curves of the four OLEDs are shown in Fig. 2a-d, and Table 2 summarizes their device performance. Although the OLEDs without assistant dopants, which use only conventional single doping by fluorescent dopants, show low device performance as shown by the open circles $\left(\eta_{\mathrm{EQE}}<5 \%\right)$, remarkably high $\eta_{\mathrm{EQE}}$ of $13.4,15.8,18$ and $17.5 \%$ for blue, green, yellow and red EL were achieved by including the assistant dopants. In addition, the magnitudes of the slopes of the $\eta_{\mathrm{EQE}}-L$ curves of the double-dopant systems are much lower than those of the corresponding single-dopant systems at similar luminance, indicating that the efficiency rolloff characteristics are well suppressed in the double-dopant system. The reduced roll-off is probably because of the expansion of the carrier recombination site or the reduction of the interaction between excitons and polarons (that is, exciton-polaron annihilation) as a result of rapid energy transfer from the carrier recombination centre (TADF assistant dopants) to the emission centre (fluorescent emitter dopants).

The EL spectra of the OLEDs are presented in Fig. 2e, Supplementary Fig. 4 and Supplementary Note 2. These OLEDs emit a full range of visible colours from blue $(462 \mathrm{~nm})$ to red $(610 \mathrm{~nm})$. Although a weak emission originating from the assistant dopant is observed in the red OLED, other OLEDs showed pure emission originating from the emitter dopants that is in good accordance with the PL spectrum of the emitter dopants in solution. Here $\Phi_{\mathrm{PL}}$ of $80 \pm 2,81 \pm 2,90 \pm 2$ and $88 \pm 2 \%$ were obtained for the blue, green, yellow and red emitter dopants when doped with assistant dopants in films (Table 1). Thus, we can estimate $\eta_{\gamma}$ of $55-84 \%, 65-97 \%, 66-100 \%$ and
66-99\%, respectively, assuming a charge carrier balance factor of 1 and a light-out-coupling efficiency of $20-30 \%$ (refs 28,29 ). These results clearly indicate that the devices overcame the theoretical limit of $25 \%$ for the singlet exciton production efficiency that is assumed for fluorescence-based OLEDs and even that of $62.5 \%$ in the case of TTA ${ }^{18}$.

Transient EL analysis. In order to confirm the contribution of singlet exciton generation via energy transfer from the assistant dopants to the emitter dopants after harvesting triplet excitons on the assistant dopants under electrical excitation, transient EL was measured with an electrical excitation pulse width of $1 \mu \mathrm{s}$ at $300 \mathrm{~K}$. Figure 3a shows the streak image and the transient EL decay curve obtained from the red OLED with an EML of 1 wt\% DBP and $15 \mathrm{wt} \%$ tri-PXZ-TRZ in a CBP host matrix. After turning off the electrical pulse excitation, a clear delayed component with emission bands centred at $610 \mathrm{~nm}$ was observed. In addition, the emission spectrum of the delayed fluorescence is consistent with that of the prompt component, which is consistent with the described mechanism of triplet to singlet exciton upconversion in tri-PXZ-TRZ followed by successive resonant singlet energy transfer to the $S_{1}^{\mathrm{E}}$ states of DBP emitters.

Here we describe the exciton formation process in the doubledopant system under either optical or electrical excitation. In the case of optical excitation, singlet excitons are mainly generated in the $S_{1}^{\mathrm{H}}$ state of a CBP host molecule by photoabsorption, and nearly all of the singlets are then resonantly transferred to the $S_{1}^{A}$ state of tri-PXZ-TRZ molecules because of the large concentration of the assistant dopants. In addition, a number of singlet excitons in tri-PXZ-TRZ would also be formed by direct absorption. Next, a fraction of the singlet excitons is transferred into the $S_{1}^{\mathrm{E}}$ state of DBP through a FRET process, producing the prompt fluorescence decay of DBP. Simultaneously, singlet excitons on tri-PXZ-TRZ also decay to the $\mathrm{T}_{1}^{\mathrm{A}}$ state internally through ICS and are successively upconverted into the $S_{1}^{A}$ state again by thermal conversion, followed by energy transfer via FRET to the $S_{1}^{\mathrm{E}}$ state of DBP that produces the delayed emission. Here we can ignore the direct formation of excitons on DBP because of the dilute concentration of $1 \mathrm{wt} \%$. Under electrical excitation, on the other hand, since the singlet and triplet excitons are directly created on the $S_{1}^{\mathrm{A}}$ and $\mathrm{T}_{1}^{\mathrm{A}}$ states of the tri-PXZ-TRZ molecules based on the exciton branching ratio of $25-75 \%$, respectively, the contribution of the delayed fluorescence in the total emission is significantly larger compared with that of the optical excitation, as shown in Fig. $3 \mathrm{~b}$. 

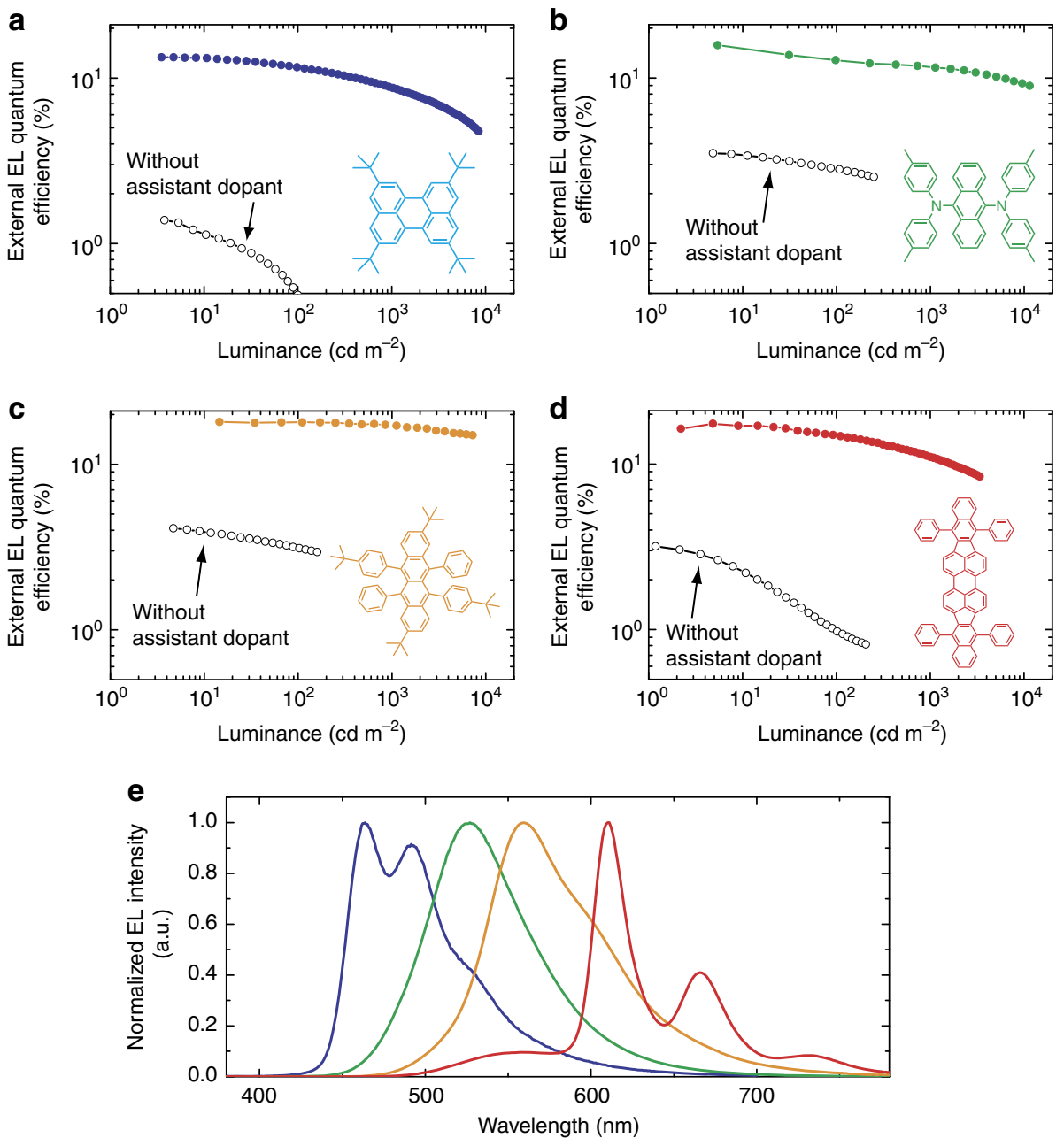

Figure 2 | Device performance of OLEDs. (a-d) External EL quantum efficiency as a function of luminance for the blue, green, yellow and red OLEDs. The external EL quantum efficiency for OLEDs without an assistant dopant is plotted as open symbols. Inset: chemical structures of emitter dopants used in this study. (e) EL spectra of the devices at a luminance of $100 \mathrm{~cd} \mathrm{~m}^{-2}$.

Table 2 | Device performance of the four colour OLEDs with assistant dopants for triplet harvesting.

\begin{tabular}{|c|c|c|c|c|c|c|c|c|c|}
\hline Device & $\begin{array}{c}\text { Turn on } \\
\text { voltage (V) }\end{array}$ & $\begin{array}{c}\text { Max EQE } \\
(\%)\end{array}$ & $\begin{array}{c}\operatorname{Max} C E \\
\left(\operatorname{cd~} A^{-1}\right)\end{array}$ & $\begin{array}{c}\text { Max PE } \\
\left(\operatorname{Im} W^{-1}\right)\end{array}$ & CIE & Voltage (V) & EQE (\%) & CE $\left(\operatorname{cd~} A^{-1}\right)$ & PE $\left(\operatorname{Im~} \mathbf{W}^{-1}\right)$ \\
\hline Green & 3.0 & 15.8 & 45 & 47 & $(0.29,0.59)$ & 4.1 & 11.7 & 38 & 30 \\
\hline Yellow & 3.2 & 18.0 & 60 & 58 & $(0.45,0.53)$ & 5.2 & 17.2 & 56 & 33 \\
\hline Red & 3.0 & 17.5 & 25 & 28 & $(0.61,0.39)$ & 6.4 & 10.9 & 20 & 10 \\
\hline
\end{tabular}

Device operational stability. The introduction of assistant dopants into a host-guest system provides not only a significant enhancement of $\eta_{\mathrm{EQE}}$ but also an enhancement of device operational stability under electrical excitation. For example, the normalized luminance of the yellow OLEDs as a function of operation time at a constant current density of $10 \mathrm{~mA} \mathrm{~cm}^{-2}$ are presented in Fig. 4. Although a rapid decrease in luminance was observed in the OLED without assistant dopants (device B), the OLED with the assistant dopants (device A) showed improved luminance decay characteristics compared with that of the OLED without the assistant dopants, resulting in an improved operational lifetime, defined as the elapsed operation time at which the luminance drops to $50 \%$ of the initial value, of $194 \mathrm{~h}$.
The driving voltages of the devices are also rather stable, displaying a rise of less than $0.5 \mathrm{~V}$ after $100 \mathrm{~h}$ of operation. More interestingly, the operational lifetime was longer than that of the OLED with PXZ-TRZ molecules as the emitter dopant (device C) while the voltage rise curves are almost the same, indicating that the combination of fluorescent emitter dopants and TADF assistant dopants provides improved device performance. We note that the nearly identical film morphology for each of the co-deposited films compared with that of the host-only films suggests that device stability is not influenced by a morphology change caused by the dopant molecules as shown in Supplementary Fig. 5 and Supplementary Note 3. Since it is well established that TBRb is a stable emitter electrochemically ${ }^{26}$, the 

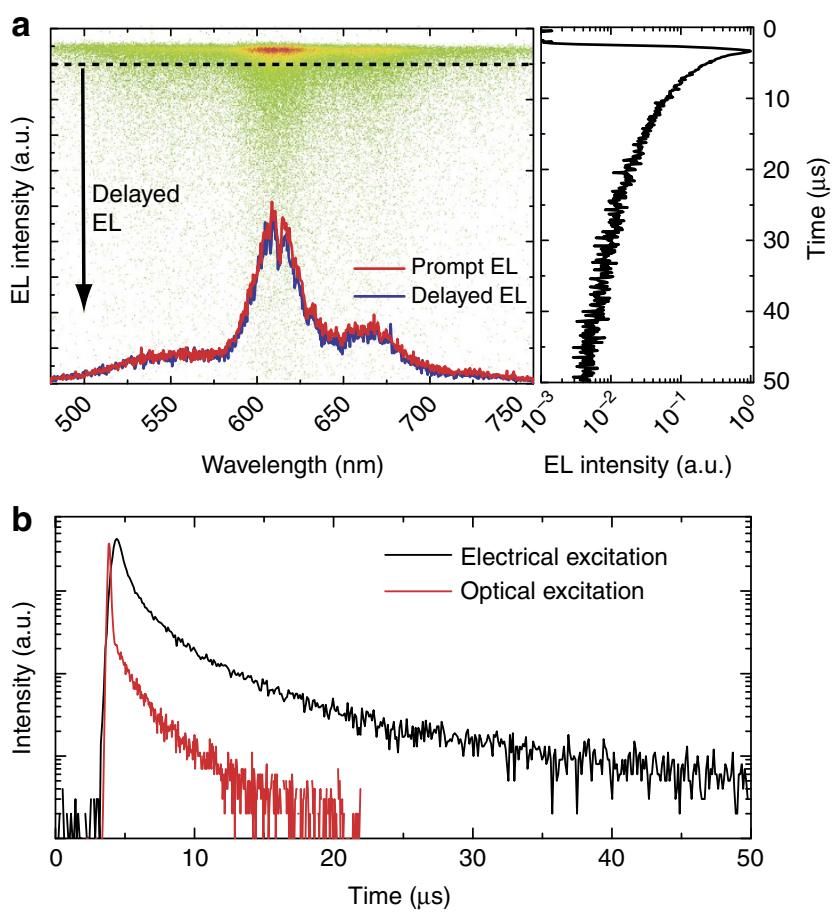

Figure 3 | Transient EL characteristics. (a) Time-resolved electroluminescence image for the red OLED. The streak image (left) and time-resolved EL decay curve (right) are for the red OLED under electrical excitation with a pulse of $1 \mu \mathrm{s}$ at $300 \mathrm{~K}$. A delayed EL spectrum was collected from 3 to $50 \mu$ s after excitation. (b) Time-resolved PL and EL response of a 1 wt\%-DBP:15 wt\%-tri-PXZ-TRZ:CBP film (red line) and 1 wt\%-DBP:15 wt\%-tri-PXZ-TRZ:CBP-based OLED (black line), respectively.

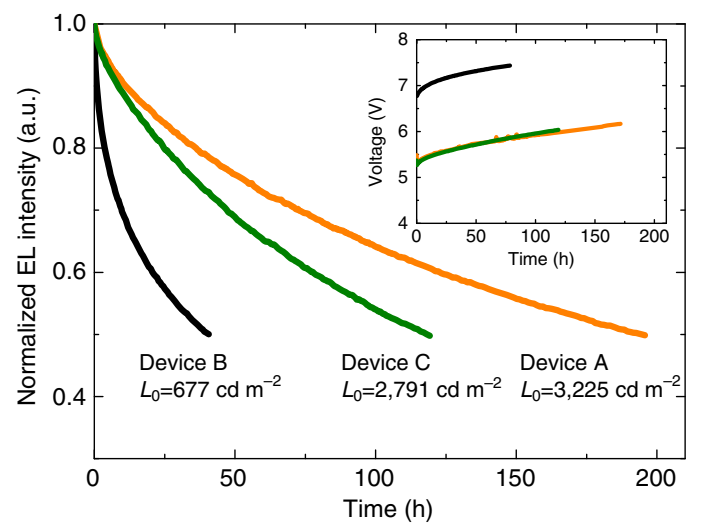

Figure 4 | Device stability of yellow OLEDs. For operational lifetime measurement of the double-dopant system, an OLED with a device structure of ITO/ $\alpha$-NPD (35 nm)/1 wt\%-TBRb:25 wt\%-PXZ-TRZ:mCBP $(30 \mathrm{~nm}) / \mathrm{T} 2 \mathrm{~T}(10 \mathrm{~nm}) / \mathrm{Alq}_{3}(55 \mathrm{~nm}) / \mathrm{LiF}(0.8 \mathrm{~nm}) / \mathrm{Al}(100 \mathrm{~nm})$ (device A) was used. To confirm the effect of the assistant dopant, OLEDs with an EML of either $1 \mathrm{wt} \%$-TBRb:mCBP (device B) or 25 wt\%-PXZ-TRZ:mCBP (device C) were also measured at a constant current density of $10 \mathrm{~mA} \mathrm{~cm}^{-2}$. Initial luminances $\left(L_{0}\right)$ are 3,225, 677 and $2,791 \mathrm{~cd} \mathrm{~m}^{-2}$ for devices $A, B$ and $C$, respectively. Inset: voltage rise curves for devices $A, B$ and $C$ (coloured accordingly).

differences in reliability would be because of the change of carrier recombination and exciton formation area with and without the presence of the assistant dopants, in addition to the electrochemical stability of emitter molecules.

\section{Discussion}

Our results suggest that both a higher $\eta_{\mathrm{EQE}}$ and an enhancement of operational stability can be expected with an optimum EML design using a wide variety of TADF and fluorescent materials. As numerous electrochemically stable fluorescent emitters have been widely developed in the past two decades, the triplet harvesting mechanism realized here can provide a greater flexibility in the design of OLED architectures. In addition, since $\eta_{\text {out }}$ can exceed $20 \%$ by using emitter molecules with horizontally oriented dipoles ${ }^{30}$, the flexibility of simple aromatic compound design can further boost the $\eta_{\mathrm{EQE}}$ by enhancement of $\eta_{\text {out }}$ without special light-out-coupling structures. In summary, we presume that the cascade energy transfer scheme using TADF assistant dopants and fluorescent emitter dopants will be the most promising device architecture for OLEDs with ultimate performance.

\section{Methods}

Materials. $\mathrm{mCP}, 4,4^{\prime}$-cyclohexylidenebis[N,N-bis(4-methylphenyl)benzenamine] (TAPC), 2,2',2'-(1,3,5-benzinetriyl)-tris(1-phenyl-1-H-benzimidazole (TPBi), TBPe, TTPA, TBRb and DBP were purchased from Luminescence Technology Corp. mCBP was purchased from NARD Institute Ltd. CBP, 4,4'-bis(N-phenyl1-naphthylamino)biphenyl ( $\alpha$-NPB) and tris(8-hydroxyquinolinato)aluminum (Alq3) were used as received from the Nippon Steel Chemical Co., Ltd. ACRSA, DPEPO $^{31}$, 2-phenoxazine-4,6-diphenyl-1,3,5-triazine (PXZ-TRX), tri-PXZ-TRZ and 2,4,6-tris(biphenyl-3-yl)-1,3,5-triazine (T2T) ${ }^{32}$ were synthesized according to the reported procedures.

Synthesis of ACRXTN. We synthesized ACRXTN according to the general procedure. The complete synthetic route for ACRXTN and the 1H NMR spectrum of ACRXTN are included in the Supplementary Methods section and Supplementary Fig. 6, respectively. NMR spectra were obtained with a Bruker Biospin Avance-III 500 NMR spectrometer at ambient temperature. High-resolution mass spectrometry by fast atom bombardment was performed using a JEOL JMS-700 spectrometer.

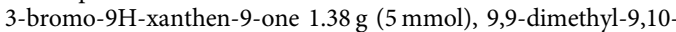
dihydroacridine $1.15 \mathrm{~g}(5.5 \mathrm{mmol})$, tert-BuONa $0.96 \mathrm{~g}(10 \mathrm{mmol})$, tri-tertbutylphosphonium tetrafluoroborate $145 \mathrm{mg}(0.5 \mathrm{mmol})$ and palladium acetate $56 \mathrm{mg}(0.25 \mathrm{mmol})$ were put into a flask and purged three times by nitrogen/ vacuum cycle. Then, anhydrous toluene was added and refluxed for $8 \mathrm{~h}$. After cooling to room temperature, the resulting solution was filtered through celite and concentrated. The crude product was purified using a silica gel chromatography $\left(\mathrm{CH}_{2} \mathrm{Cl}_{2}\right.$ :hexane $\left.=8: 2\right)$ and recrystallized twice from mixed solvent of hexane:AcOEt $=9: 1 \mathrm{ml} \mathrm{g}^{-1}$. The desired product was obtained as yellow powder $(2.06 \mathrm{~g}, 85 \%) .{ }^{1} \mathrm{H}-\mathrm{NMR}\left(500 \mathrm{MHz}, \mathrm{CDCl}_{3}\right): \delta$ (p.p.m.) $=8.53(\mathrm{~d}, 1 \mathrm{H}, 8.5 \mathrm{~Hz}), 8.38$ (dd, $1 \mathrm{H}, 8 \mathrm{~Hz}), 7.74$ (ddd, $1 \mathrm{H}, 8.6,7.2,1.8 \mathrm{~Hz}), 7.53(\mathrm{~d}, 1 \mathrm{H}, 1.9 \mathrm{~Hz}), 7.52-7.46$ (m, 3H), 7.42 (ddd, $1 \mathrm{H}, 8.1,7.2,1 \mathrm{~Hz}), 7.38(\mathrm{dd}, 1 \mathrm{H}, 8.5,2 \mathrm{~Hz}), 7.08-6.98(\mathrm{~m}, 4 \mathrm{H})$, 6.53 (dd, $2 \mathrm{H}, 7.8,1.6 \mathrm{~Hz}) .{ }^{13} \mathrm{C}-\mathrm{NMR}\left(125 \mathrm{MHz}, \mathrm{CDCl}_{3}\right): \delta$ (p.p.m.) $=176.47$, $157.83,156.25,148.09,140.28,134.94,132.55,129.32,126.79,126.45,125.27$, $124.59,124.20,121.99,121.96,120.52,117.95,117.57,115.88,36.38,30.53$. Highresolution mass spectrometry $(\mathrm{m} / \mathrm{z}):[\mathrm{M}+\mathrm{H}]+$ calculated for $\mathrm{C} 28 \mathrm{H} 22 \mathrm{NO} 2$, 404.1651; found, 404.1651.

Optical characterization of organic thin films. PL quantum efficiency was measured by an absolute PL quantum yield measurement system (C11347-01, Hamamatsu Photonics) under the flow of nitrogen gas with an excitation wavelength of $337 \mathrm{~nm}$. Low-temperature PL intensity and emission lifetimes were measured using a streak camera (C4334, Hamamatsu Photonics) and cryostat (Iwatani Industrial Gases Co.) with a nitrogen gas laser (MNL200, Laser Technik) as an excitation light source under a pressure of about $3 \mathrm{~Pa}$.

Fabrication of OLEDs. Glass substrates with a pre-patterned, 100-nm-thick, $100 \mathrm{Ohm} \mathrm{sq}^{-2}$ tin-doped indium oxide (ITO) coating were used as anodes. Substrates were washed by sequential ultrasonication in neutral detergent, distilled water, acetone and isopropanol, and then exposed to ultraviolet-ozone (NLUV253, Nippon Laser \& Electronics Lab) to remove adsorbed organic species. After pre-cleaning of the substrates, effective device areas of $1 \mathrm{~mm}^{2}$ were defined on the patterned-ITO substrates by a polyimide insulation layer using a conventional photolithography technique. Substrates were treated with ultraviolet-ozone for $25 \mathrm{~min}$ and immediately transferred into the evaporation chamber.

Organic layers were formed by thermal evaporation. Doped emitting layers were deposited by co-evaporation. Deposition was performed under vacuum at pressures of $<5 \times 10^{-5} \mathrm{~Pa}$. Devices were exposed to nitrogen gas once after formation of the organic layers to apply a metal mask that defines the cathode area After device fabrication, devices were immediately encapsulated with glass lids 
using epoxy glue in a nitrogen-filled glove box $\left(\mathrm{O}_{2}<0.1\right.$ p.p.m., $\mathrm{H}_{2} \mathrm{O}<0.1$ p.p.m. $)$. Commercial calcium oxide desiccant (Dynic Co.) was included in each encapsulated package.

Blue OLEDs with the structure ITO/ $\alpha$-NPB $(35 \mathrm{~nm}) / \mathrm{mCP}(10 \mathrm{~nm}) / 1 \mathrm{wt} \%$ TBPe: $15 \mathrm{wt} \%$-ACRSA: DPEPO $(15 \mathrm{~nm}) / \mathrm{DPEPO}(8 \mathrm{~nm}) / \mathrm{TPBi}(57 \mathrm{~nm}) / \mathrm{LiF}$ $(0.8 \mathrm{~nm}) / \mathrm{Al}(100 \mathrm{~nm})$ were fabricated. As a control device, an OLED with an EML that consisted of $1 \mathrm{wt} \%$-TBPe: DPEPO was also made.

Green OLEDs with the structure ITO/TAPC $(35 \mathrm{~nm}) / 1 \mathrm{wt} \%$-TTPA: $50 \mathrm{wt} \%$ ACRXTN: $\mathrm{mCP}(15 \mathrm{~nm}) / \mathrm{TPBi}(65 \mathrm{~nm}) / \mathrm{LiF}(0.8 \mathrm{~nm}) / \mathrm{Al}(100 \mathrm{~nm})$ were fabricated. As a control device, an OLED with an EML consisting of $1 \mathrm{wt} \%$-TTPA: $\mathrm{mCP}$ was also made.

Yellow OLEDs with the structure ITO/TAPC $(35 \mathrm{~nm}) / 1 \mathrm{wt} \%$-TBRb:25 wt\%PXZ-TRX:mCBP $(30 \mathrm{~nm}) / \mathrm{T} 2 \mathrm{~T}(10 \mathrm{~nm}) / \mathrm{Alq}_{3}(55 \mathrm{~nm}) / \mathrm{LiF}(0.8 \mathrm{~nm}) / \mathrm{Al}(100 \mathrm{~nm})$ were fabricated. As a control device, an OLED with an EML consisting of $1 \mathrm{wt} \%$-TBRb: mCBP was also made.

Red OLEDs with the structure ITO/TAPC (35 nm)/1 wt\%-DBP: $15 \mathrm{wt} \%$-triPXZ-TRZ:CBP $(15 \mathrm{~nm}) / \mathrm{TPBi}(65 \mathrm{~nm}) / \mathrm{LiF}(0.8 \mathrm{~nm}) / \mathrm{Al}(100 \mathrm{~nm})$ were fabricated. As a control device, an OLED with an EML consisting of $1 \mathrm{wt} \%$-DBP: CBP was also made.

Schematic diagrams of the energy levels of the fabricated devices and the chemical structures of the assistant dopant materials used in them are presented in Supplementary Fig. 7.

Characterization of OLEDs. The current density-voltage-luminance characteristics of the OLEDs were evaluated using a source meter (Keithley 2400, Keithley Instruments Inc.) and an absolute external quantum efficiency measurement system (C9920-12, Hamamatsu Photonics). The OLEDs were mounted to the entrance port of the measurement system's integrating sphere to collect the photons emitted from the front face of the devices. Each EL spectrum was collected by an optical fiber connected to a spectrometer (PMA-12, Hamamatsu Photonics). The repeatability of device performances of the present devices was confirmed by four different samples. To confirm the validity of our $\eta_{\mathrm{EOE}}$ measurements, we also measured $\eta_{\mathrm{EQE}}$ using another independent measurement system based on a luminance meter (Supplementary Fig. 8 and Supplementary Note 4). Time-resolved EL decay curves were obtained using a streak camera (C4334, Hamamatsu Photonics) with a pulse generator (81101A, Agilent) as an electrical excitation source. The operational lifetime was measured using a luminance meter (SR-3AR, TOPCON) at a constant DC current. All measurements were performed in ambient atmosphere at room temperature.

\section{References}

1. Aldo, M. A., O'Brien, D. F., Thompson, M. E. \& Forrest, S. R. Excitonic singlet-triplet ratio in a semiconducting organic thin film. Phys. Rev. B 60 , 14422-14428 (1999)

2. Tsutsui, T., Adachi, C. \& Saito, S. in Photochemical Processes in Organized Molecular Systems. (ed. Honda, K.) 437-450 (Elsevier, 1991).

3. Kido, J., Nagai, K. \& Ohashi, Y. Electroluminescence in a terbium complex. Chem. Lett. 19, 657-660 (1990).

4. Kido, J., Hayase, H., Hongawa, K., Nagai, K. \& Okuyama, K. Bright red lightemitting organic electroluminescent devices having a europium complex as an emitter. Appl. Phys. Lett. 65, 2124 (1994).

5. Hong, Z. R. et al. Rare earth complex as a high-efficiency emitter in an electroluminescent device. Adv. Mater. 13, 1241-1245 (2001).

6. Baldo, M. A. et al. Highly efficient phosphorescent emission from organic electroluminescent devices. Nature 395, 151-154 (1998).

7. Baldo, M. A., Lamansky, S., Burrows, P. E., Thompson, M. E. \& Forrest, S. R. Very high-efficiency green organic light-emitting devices based on electrophosphorescence. Appl. Phys. Lett. 75, 4-6 (1999).

8. Adachi, C., Baldo, M. A., Thompson, M. E. \& Forrest, S. R. Nearly $100 \%$ internal phosphorescence efficiency in an organic light-emitting device. J. Appl. Phys. 90, 5048-5050 (2001).

9. Watanabe, S., Ide, N. \& Kido, J. High-efficiency green phosphorescent organic light-emitting devices with chemically doped layers. Jpn J. Appl. Phys. 46, 11861188 (2007).

10. Reineke, S. et al. White organic light-emitting diodes with fluorescent tube efficiency. Nature 459, 234-238 (2009).

11. Baldo, M. A., Thompson, M. E. \& Forrest, S. R. High-efficiency fluorescent organic light-emitting devices using a phosphorescent sensitizer. Nature 403, 750-753 (2000)

12. D'Andrade, B. W. et al. High-efficiency yellow double-doped organic light-emitting devices based on phosphor-sensitized fluorescence. Appl. Phys. Lett. 79, 1045-1047 (2001).

13. Iwakuma, T. et al. Red and white EL materials based on a new fused aromatic ring. SID Int. Symp. Digest Tech. Papers 33, 598-601 (2002).

14. Hosokawa, C. et al. Improvement of lifetime in organic electroluminescence. SID Int. Symp. Digest Tech. Papers 35, 780-783 (2004).

15. Kawamura, M. et al. Highly efficient fluorescent blue OLEDs with efficiencyenhancement layer. SID Int. Symp. Digest Tech. Papers 41, 560-563 (2010).
16. Helfrich, W. \& Schneider, W. G. Transients of volume-controlled current and of recombination radiation in anthracene. J. Chem. Phys. 44, 2902-2909 (1966).

17. Pope, M. \& Swenberg, C. E. Electronic Processes in Organic Crystals 64 (Oxford University Press, 1982)

18. Kondakov, D. Y., Pawlik, T. D., Hatwar, T. K. \& Spindler, J. P. Triplet annihilation exceeding spin statistical limit in highly efficient fluorescent organic light-emitting diodes. J. Appl. Phys. 106, 124510-124516 (2009).

19. Endo, A. et al. Thermally activated delayed fluorescence from $\mathrm{Sn}^{4+}$-porphyrin complexes and their application to organic light emitting diodes-a novel mechanism for electroluminescence. Adv. Mater. 21, 4802-4806 (2009).

20. Tanaka, H., Shizu, K., Miyazaki, H. \& Adachi, C. Efficient green thermally activated delayed fluorescence (TADF) from a phenoxazine-triphenyltriazine (PXZ-TRZ) derivative. Chem. Commun. 48, 11392-11394 (2012).

21. Uoyama, H., Goushi, K., Shizu, K., Nomura, H. \& Adachi, C. Highly efficient organic light-emitting diodes from delayed fluorescence. Nature 492, 234-238 (2012).

22. Tanaka, H., Shizu, K., Nakanotani, H. \& Adachi, C. Twisted intramolecular charge transfer state for long-wavelength thermally activated delayed fluorescence. Chem. Mater. 25, 3766-3771 (2013).

23. Nasu, K. et al. A highly luminescent spiro-anthracenone-based organic light-emitting diode through thermally activated delayed fluorescence. Chem. Commun. 49, 10385-10387 (2013).

24. Mi, B.-X. et al. Reduction of molecular aggregation and its application to the high-performance blue perylene-doped organic electroluminescent device. Appl. Phys. Lett. 75, 4055-4057 (1999).

25. Yu, Y.-H., Huang, C.-H., Yeh, J.-M. \& Huang, P.-T. Effect of methyl substituents on the $\mathrm{N}$-diaryl rings of anthracene-9,10-diamine derivatives for OLEDs applications. Org. Electron 12, 694-702 (2011).

26. Wu, Y.-S., Liu, T.-H., Chen, H.-H. \& Chen, C.-H. A new yellow fluorescent dopant for high-efficiency organic light-emitting devices. Thin Solid Films 496, 626-630 (2006).

27. Okumoto, K., Kanno, H., Hamada, Y., Takahashi, H. \& Shibata, K. High efficiency red organic light-emitting devices using tetraphenyldibenzoperiflanthene-doped rubrene as an emitting layer. Appl. Phys. Lett. 89, 013502-013504 (2006).

28. Smith, L. H., Wasey, J. A. E. \& Barnes, W. L. Light out coupling efficiency of top-emitting organic light-emitting diodes. Appl. Phys. Lett. 84, 2986-2988 (2004).

29. Tanaka, D. et al. Ultra high efficiency green organic light-emitting devices. Jpn J. Appl. Phys. 46, L10-L12 (2007).

30. Yokoyama, D. Molecular orientation in small-molecule organic light-emitting diodes. J. Mater. Chem. 21, 19187-19202 (2011).

31. Han, C. et al. A simple phosphine-oxide host with a multi-insulating structure: high triplet energy level for efficient blue electrophosphorescence. Chem.Eur. J. 17, 5800-5803 (2011).

32. Chen, H.-F. et al. 1,3,5-Triazine derivatives as new electron transport-type host materials for highly efficient green phosphorescent OLEDs. J. Mater. Chem. 19, 8112-8118 (2009).

\section{Acknowledgements}

This work was supported in part by the Funding Program for World-Leading Innovative R\&D on Science and Technology (FIRST) and the International Institute for Carbon Neutral Energy Research (WPI-I2CNER) sponsored by the Ministry of Education, Culture, Sports, Science and Technology (MEXT). We thank Ms Nozomi Nakamura and Mr Hiroshi Miyazaki for synthesis and purification of organic materials. We also thank W. Potscavage for his assistance with preparation of this manuscript.

\section{Author contributions}

The experiments were conceived and designed by H.N. and K.M. and carried out by H.N., T.H., T.F. and K.M. M.N., H.T., Y.S. and T.Y. performed the synthetic work H.N. and C.A. wrote the manuscript. The project was supervised by C.A. All the authors discussed the results and contributed to the article.

\section{Additional information}

Supplementary Information accompanies this paper at http://www.nature.com/ naturecommunications

Competing financial interests: The authors declare no competing financial interests.

Reprints and permission information is available online at http://npg.nature.com/ reprintsandpermissions/

How to cite this article: Nakanotani, H. et al. High-efficiency organic light-emitting diodes with fluorescent emitters. Nat. Commun. 5:4016 doi: 10.1038/ncomms5016 (2014). 